\title{
On the Stark broadening of some Cr II spectral lines in plasma
}

\author{
A. Almodlej ${ }^{1}$, N. Alwadie ${ }^{1,2}$, N. Ben Nessib ${ }^{1,3}$ and \\ M. S. Dimitrijević $\dot{c}^{4,5}$ \\ ${ }^{1}$ Department of Physics and Astronomy, College of Sciences, King Saud \\ University, Saudi Arabia \\ 2 Department of Physics, College of Sciences, King Khalid University, Saudi \\ Arabia \\ ${ }^{3}$ GRePAA, INSAT, Centre Urbain Nord, University of Carthage, Tunis, \\ Tunisia \\ 4 Astronomical Observatory, Volgina 7, 11060 Belgrade 38, Serbia \\ 5 Sorbonne Université, Observatoire de Paris, Université PSL, CNRS, \\ LERMA, F-92190 Meudon, France
}

Received: July 31, 2019; Accepted: September 28, 2019

\begin{abstract}
New electron-impact line widths for eight Cr II multiplets have been calculated within the modified semiempirical (MSE) approach. Needed energy levels and radial integrals are calculated by different methods. The Stark widths are obtained as a function of temperature, for perturber density of $10^{17} \mathrm{~cm}^{-3}$ and have been compared with the approximate formula of Cowley and with recent experimental results. The obtained results are of interest for diagnostic and modeling of laboratory and stellar plasmas. The obtained data will be included in the STARK-B database, which is part of the Virtual Atomic and Molecular Data Center VAMDC.
\end{abstract}

Key words: Spectral lines - Plasma - Atomic data - Stark broadening

\section{Introduction}

Chromium in various ionization stages has been observed in the spectra of white dwarf atmospheres, where the Stark broadening is usually dominant broadening mechanism, especially in deeper atmospheric layers (Simić et al., 2006; Hamdi et al., 2008; Dufour et al., 2011; Hamdi et al., 2014; Dimitrijević and Chougule, 2018). In order to determine chromium abundance in such stars, as well as for opacity calculations and more sophysticated stellar atmosphere modeling, we need the corresponding Stark broadening data. Such data are also of interest for laboratory plasma diagnostics and modeling, as well as for investigation of fusion and laser produced plasmas where addition of different impurities may change different plasma parameters.

Nine Cr I spectral line widths, due to Stark broadening, are calculated by Dimitrijević et al. (2005). Seven multiplets belonging to 4s-4p transitions of $\mathrm{Cr}$ 
II spectral lines are calculated by Dimitrijević et al. (2007) and nine resonant Cr II multiplets by Simić et al. (2013). Stark spectral line widths of six Cr III transitions are calculated by Dimitrijević and Chougule (2018). Cr VI spectral line widths for two multiplets are calculated by Dimitrijević et al. (2017). In this work, in order to provide new Stark broadening data for stellar and laboratory plasma research, we calculate Stark spectral line widths for eight Cr II multiplets for an electron density of $10^{17} \mathrm{~cm}^{-3}$ and a range of temperature between $5000 \mathrm{~K}$ and $50000 \mathrm{~K}$. The obtained results will be in STARK-B database (http://starkb.obspm.fr/, Sahal-Bréchot et al. (2015)) which is included in Virtual Atomic and Molecular Data Center (VAMDC Consortium http://vamdc.org Dubernet et al. (2016), Moreau et al. (2018)).

\section{Method of calculations}

The modified semiempirical approach (MSE) by Dimitrijević and Konjević (1980) has its origin from the semiempirical approach (SE) by Griem (1968) where Stark widths are calculated as a function of inelastic cross sections for the emitter collisions with perturbers, expressed using the empirical Gaunt factors $g(x)$, within the impact approximation taking into account the elastic collisions approximately, by extrapolation of Gaunt factors below the threshold for inelastic collisions:

$$
W_{S E}=N \frac{8 \pi}{3} \frac{\hbar^{2}}{m^{2}}\left(\frac{2 m}{\pi k T}\right)^{1 / 2} \frac{\pi}{\sqrt{3}}\left[\sum_{i^{\prime}} R_{i^{\prime} i}^{2} g\left(\frac{E}{\Delta E_{i^{\prime} i}}\right)+\sum_{f^{\prime}} R_{f^{\prime} f}^{2} g\left(\frac{E}{\Delta E_{f^{\prime} f}}\right)\right]
$$

where $\Delta E=\left|E-E_{j}\right|$.

In the MSE approach (Dimitrijević and Konjević, 1980) a modified Gaunt factors $\tilde{g}(x)=0.7-\frac{1.1}{Z}+g(x)$ is used for $\Delta \mathrm{n}=0$ :

$$
\begin{gathered}
W_{M S E}=N \frac{8 \pi}{3} \frac{\hbar^{2}}{m^{2}}\left(\frac{2 m}{\pi k T}\right)^{1 / 2} \frac{\pi}{\sqrt{3}}\left[R_{l_{i}, l_{i}+1}^{2} \tilde{g}\left(\frac{E}{\Delta E_{l_{i}, l_{i}+1}}\right)\right. \\
+R_{l_{i}, l_{i}-1}^{2} \tilde{g}\left(\frac{E}{\Delta E_{l_{i}, l_{i}-1}}\right) \\
+R_{l_{f}, l_{f}+1}^{2} \tilde{g}\left(\frac{E}{\Delta E_{l_{f}, l_{f}+1}}\right)+R_{l_{f}, l_{f}-1}^{2} \tilde{g}\left(\frac{E}{\Delta E_{l_{f}, l_{f}-1}}\right) \\
\left.+\sum_{i^{\prime}}\left(R_{i i^{\prime}}^{2}\right)_{\Delta n \neq 0} g\left(\frac{3 k T T_{i}^{* 3}}{4 Z^{2} E_{H}}\right)+\sum_{f^{\prime}}\left(R_{f f^{\prime}}^{2}\right)_{\Delta n \neq 0} g\left(\frac{3 k T n_{f}^{* 3}}{4 Z^{2} E_{H}}\right)\right]
\end{gathered}
$$

where $Z-1$ is the ionic charge and $n_{i}^{*}$ and $n_{f}^{*}$ are the effective principal quantum numbers of initial and final transition level respectively (in its original form, the spectral line width depends only on the upper principal quantum number). The effective principal quantum number $n_{j}^{*}$ is related by the energy level $E_{j}$ as:

$$
\left(n_{j}^{*}\right)^{2}=Z^{2} \frac{E_{H}}{E_{i o n}-E_{j}}
$$

where $E_{H}$ is the hydrogen atom energy. 
Energy levels needed in our MSE calculations are taken from the NIST database (Kramida et al., 2018), oscillator strengths were calculated using the method of Bates and Damgaard (1949) and tables of Oertel and Shomo (1968). For higher levels, the method described in van Regemorter et al. (1979) was applied.

The MSE is valid for singly and multicharged ions and the number of input atomic data is minimized. In the MSE approach, only the matrix elements of transitions with $\Delta \mathrm{n}=0$ should be calculated, while other transitions are separated and grouped together. The advantage of this method is that smaller number of atomic data is needed for calculation than the more sophisticated calculations in semiclassical perturbation (SCP) ones (Sahal-Bréchot et al., 2014). Accuracy of MSE results is usually within an error bars of \pm 50 percent.

The Cowley formula (Cowley, 1971) was used extensively in astrophysics, for example it was used by Majlinger et al. (2017):

$$
W_{\text {Cowley }}=N \frac{\pi}{c} \frac{\hbar^{2}}{m^{2}}\left(\frac{2 m}{\pi k T}\right)^{1 / 2} \frac{\lambda^{2}}{Z^{2}}\left[\left(n_{i}^{*}\right)^{4}+\left(n_{f}^{*}\right)^{4}\right]
$$

where $\lambda$ is the wavelength of the line.

\section{Results and discussion}

We evaluated Stark widths using the MSE approach for 8 selected Cr II spectral lines. The Stark widths are calculated for an electron density of $10^{17} \mathrm{~cm}^{-3}$, which is usually used in tables for ion emitters, and for different temperature values. To make the usage of obtained results for modelisation of stellar atmospheres and spectra easier, as well as for radiative transfer calculations, and to prepare our results for the implementation in the STARK-B database, we used the formula from Sahal-Bréchot et al. (2011) for fitting calculated data within the range 5 $000 \mathrm{~K} 50000 \mathrm{~K}$ with temperature:

$$
\log (W)=a_{0}+a_{1} \log (T)+a_{2} \log ^{2}(T)
$$

where, $W$ is the FWHM Stark width in $\AA, T$ is the temperature in Kelvin and $a_{0}, a_{1}$ and $a_{2}$ are the fitting parameters obtained for electron density of $10^{17}$ $\mathrm{cm}^{-3}$.

Stark width (FWHM) values for temperatures from $5000 \mathrm{~K}$ to $50000 \mathrm{~K}$ are given in Table 1, since this temperature interval is interesting for plasma diagnostic of white dwarf atmospheres and laser produced plasmas.

In this Table 1, $\mathrm{W}_{M S E}$ is our calculated Stark width using the MSE approach (Equation 1) and $\mathrm{W}_{\text {Cowley }}$ using Equation 4. The wavelengths in Table 1 are calculated from the corresponding energy levels given as the input so that they may differ from the observed ones.

We give in Table 2 , the fitting parameters $a_{0}, a_{1}$ and $a_{2}$ with the $R$ correlation coefficient in $\%$. 
Table 1. Stark electron-impact widths (full widths at half maximum, FWHM) for Cr II spectral lines: $\mathrm{W}_{M S E}$, calculated by using the modified semiempirical (MSE) method; $\mathrm{W}_{\text {Cowley }}$, calculated by using the approximate formula of Cowley. The temperatures are from $5000 \mathrm{~K}$ up to $50000 \mathrm{~K}$ and the electron density is $10^{17} \mathrm{~cm}^{-3}$.

\begin{tabular}{|c|c|c|c|}
\hline Transition & Temp. & $\mathrm{W}_{M S E}$ & $\mathrm{~W}_{\text {Cowley }}$ \\
\hline \multirow[t]{2}{*}{ Cr II $3 \mathrm{~d}^{4}\left(\mathrm{a}^{1} \mathrm{G}\right) 4 \mathrm{~s}^{2} \mathrm{P}-3 \mathrm{~d}^{4}\left(\mathrm{a}^{1} \mathrm{G}\right) 4 \mathrm{p}^{2} \mathrm{P}^{o}$} & 5000 & $1.58 \mathrm{E}-01$ & $1.65 \mathrm{E}-01$ \\
\hline & 10000 & $1.12 \mathrm{E}-01$ & $1.17 \mathrm{E}-01$ \\
\hline $3172.07 \AA$ & 20000 & 7.92E-02 & $8.26 \mathrm{E}-02$ \\
\hline \multirow{2}{*}{$3 \mathrm{kT} / 2 \Delta \mathrm{E}=0.349$} & 30000 & $6.47 \mathrm{E}-02$ & $6.75 \mathrm{E}-02$ \\
\hline & 50000 & $5.01 \mathrm{E}-02$ & $5.23 \mathrm{E}-02$ \\
\hline \multirow[t]{2}{*}{ Cr II $3 d^{4}\left(a^{3} F\right) 4 s^{2} F-3 d^{4}\left(a^{3} F\right) 4 p^{2} F^{o}$} & 5000 & $1.43 \mathrm{E}-01$ & $152 \mathrm{E}-01$ \\
\hline & 10000 & $1.01 \mathrm{E}-01$ & $1.08 \mathrm{E}-01$ \\
\hline & 20000 & $7.16 \mathrm{E}-02$ & $7.62 \mathrm{E}-02$ \\
\hline \multirow{2}{*}{$3 \mathrm{kT} / 2 \Delta \mathrm{E}=1.14$} & 30000 & $5.84 \mathrm{E}-02$ & $6.22 \mathrm{E}-02$ \\
\hline & 50000 & $4.53 \mathrm{E}-02$ & $4.82 \mathrm{E}-02$ \\
\hline \multirow[t]{2}{*}{ Cr II $3 d^{4}\left({ }^{3} G\right) 4 s^{2} G-3 d^{4}\left({ }^{3} G\right) 4 p^{2} G^{o}$} & 5000 & $1.55 \mathrm{E}-01$ & $1.65 \mathrm{E}-01$ \\
\hline & 10000 & $1.09 \mathrm{E}-01$ & $1.17 \mathrm{E}-01$ \\
\hline \multirow{3}{*}{$\begin{array}{c}3107.56 \AA \\
3 \mathrm{kT} / 2 \Delta \mathrm{E}=0.327\end{array}$} & 20000 & $7.73 \mathrm{E}-02$ & $8.27 \mathrm{E}-02$ \\
\hline & 30000 & $6.31 \mathrm{E}-02$ & $6.75 \mathrm{E}-02$ \\
\hline & 50000 & 4.89E-02 & $5.23 \mathrm{E}-02$ \\
\hline \multirow[t]{2}{*}{ Cr II $3 d^{4}\left(a^{1} G\right) 4 s^{2} G-3 d^{4}\left(a^{1} G\right) 4 p^{2} G^{o}$} & 5000 & $1.17 \mathrm{E}-01$ & $1.27 \mathrm{E}-01$ \\
\hline & 10000 & 8.24 & $9.01 \mathrm{E}-02$ \\
\hline & 20000 & $5.83 \mathrm{E}-02$ & $6.37 \mathrm{E}-02$ \\
\hline \multirow{2}{*}{$3 \mathrm{kT} / 2 \Delta \mathrm{E}=0.302$} & 30000 & $4.76 \mathrm{E}-02$ & $5.20 \mathrm{E}-02$ \\
\hline & 50000 & $3.69 \mathrm{E}-02$ & $4.03 \mathrm{E}-02$ \\
\hline \multirow[t]{2}{*}{ Cr II $3 d^{4}\left(a^{3} P\right) 4 s^{4} P-3 d^{4}\left(a^{3} P\right) 4 p^{4} D^{o}$} & 5000 & $1.26 \mathrm{E}-01$ & $1.34 \mathrm{E}-01$ \\
\hline & 10000 & $8.92 \mathrm{E}-02$ & $9.47 \mathrm{E}-02$ \\
\hline & 20000 & $6.31 \mathrm{E}-02$ & $6.69 \mathrm{E}-02$ \\
\hline \multirow{2}{*}{$3 \mathrm{kT} / 2 \Delta \mathrm{E}=0.307$} & 30000 & $5.15 \mathrm{E}-02$ & $5.47 \mathrm{E}-02$ \\
\hline & 50000 & $3.99 \mathrm{E}-02$ & $4.23 \mathrm{E}-02$ \\
\hline \multirow[t]{2}{*}{ Cr II $3 \mathrm{~d}^{4}\left({ }^{3} \mathrm{H}\right) 4 \mathrm{~s}^{4} \mathrm{H}-3 \mathrm{~d}^{4}\left({ }^{3} \mathrm{H}\right) 4 \mathrm{p}^{4} \mathrm{H}^{\circ}$} & 5000 & $1.28 \mathrm{E}-01$ & $1.36 \mathrm{E}-01$ \\
\hline & 10000 & $9.07 \mathrm{E}-02$ & $9.61 \mathrm{E}-02$ \\
\hline $2981.09 \AA$ & 20000 & $6.41 \mathrm{E}-02$ & $6.80 \mathrm{E}-02$ \\
\hline \multirow[t]{2}{*}{$3 \mathrm{kT} / 2 \Delta \mathrm{E}=0.311$} & 30000 & $5.24 \mathrm{E}-02$ & $5.55 \mathrm{E}-02$ \\
\hline & 50000 & $4.06 \mathrm{E}-02$ & 4.30E-02 \\
\hline \multirow[t]{2}{*}{ Cr II $3 \mathrm{~d}^{4}\left({ }^{3} \mathrm{D}\right) 4 \mathrm{~s}^{4} \mathrm{D}-3 \mathrm{~d}^{4}\left({ }^{3} \mathrm{D}\right) 4 \mathrm{p}^{4} \mathrm{D}^{o}$} & 5000 & $1.17 \mathrm{E}-01$ & $1.26 \mathrm{E}-01$ \\
\hline & 10000 & $8.28 \mathrm{E}-02$ & $8.92 \mathrm{E}-02$ \\
\hline $2846.26 \AA$ & 20000 & $5.85 \mathrm{E}-02$ & $6.31 \mathrm{E}-02$ \\
\hline \multirow[t]{2}{*}{$3 \mathrm{kT} / 2 \Delta \mathrm{E}=0.297$} & 30000 & $4.78 \mathrm{E}-02$ & $5.15 \mathrm{E}-02$ \\
\hline & 50000 & $3.70 \mathrm{E}-02$ & $3.99 \mathrm{E}-02$ \\
\hline \multirow[t]{2}{*}{ Cr II $3 \mathrm{~d}^{4}\left({ }^{5} \mathrm{D}\right) 4 \mathrm{~s}^{6} \mathrm{D}-3 \mathrm{~d}^{4}\left({ }^{5} \mathrm{D}\right) 4 \mathrm{p}^{6} \mathrm{~F}^{o}$} & 5000 & $1.17 \mathrm{E}-01$ & $1.26 \mathrm{E}-01$ \\
\hline & 10000 & $8.26 \mathrm{E}-02$ & $8.89 \mathrm{E}-02$ \\
\hline & 20000 & $5.84 \mathrm{E}-02$ & $6.29 \mathrm{E}-02$ \\
\hline \multirow{2}{*}{$3 \mathrm{kT} / 2 \Delta \mathrm{E}=0.297$} & 30000 & 4.77E-02 & $5.13 \mathrm{E}-02$ \\
\hline & 50000 & $3.70 \mathrm{E}-02$ & $3.98 \mathrm{E}-02$ \\
\hline
\end{tabular}


Table 2. Fitting parameters for approximate formula (5). $\mathrm{R}^{2}(\%)$ is the square of the correlation coefficient.

\begin{tabular}{cccccc}
\hline \hline Transition & $\lambda(\AA)$ & $a_{0}$ & $a_{1}$ & $a_{2}$ & $\mathrm{R}^{2}(\%)$ \\
\hline $3 \mathrm{~d}^{4}\left(\mathrm{a}^{1} \mathrm{G}\right) 4 \mathrm{~s}^{2} \mathrm{P}-3 \mathrm{~d}^{4}\left(\mathrm{a}^{1} \mathrm{G}\right) 4 \mathrm{p}^{2} \mathrm{P}^{o}$ & 3172.07 & 1.00340 & -0.47920 & -0.00235 & 0.9999976 \\
$3 \mathrm{~d}^{4}\left(\mathrm{a}^{3} \mathrm{~F}\right) 4 \mathrm{~s}^{2} \mathrm{~F}-3 \mathrm{~d}^{4}\left(\mathrm{a}^{3} \mathrm{~F}\right) 4 \mathrm{p}^{2} \mathrm{~F}^{o}$ & 3028.12 & 1.02325 & -0.50967 & 0.00126 & 0.9999966 \\
$3 \mathrm{~d}^{4}\left({ }^{3} \mathrm{G}\right) 4 \mathrm{~s}^{2} \mathrm{G}-3 \mathrm{~d}^{4}\left({ }^{3} \mathrm{G}\right) 4 \mathrm{p}^{2} \mathrm{G}^{o}$ & 3107.56 & 1.12564 & -0.54132 & 0.00487 & 0.9999823 \\
$3 \mathrm{~d}^{4}\left(\mathrm{a}^{1} \mathrm{G}\right) 4 \mathrm{~s}^{2} \mathrm{G}-3 \mathrm{~d}^{4}\left(\mathrm{a}^{1} \mathrm{G}\right) 4 \mathrm{p}^{2} \mathrm{G}^{o}$ & 2777.27 & 0.99869 & -0.53851 & 0.00448 & 0.9999913 \\
$3 \mathrm{~d}^{4}\left(\mathrm{a}^{3} \mathrm{P}\right) 4 \mathrm{~s}^{4} \mathrm{P}-3 \mathrm{~d}^{4}\left(\mathrm{a}^{3} \mathrm{P}\right) 4 \mathrm{p}^{4} \mathrm{D}^{o}$ & 2943.75 & 0.92591 & -0.48882 & -0.00127 & 0.9999993 \\
$3 \mathrm{~d}^{4}\left({ }^{3} \mathrm{H}\right) 4 \mathrm{~s}^{4} \mathrm{H}-3 \mathrm{~d}^{4}\left({ }^{3} \mathrm{H}\right) 4 \mathrm{p}^{4} \mathrm{H}^{o}$ & 2981.09 & 0.93937 & -0.49250 & -0.00075 & 0.9999985 \\
$3 \mathrm{~d}^{4}\left({ }^{3} \mathrm{D}\right) 4 \mathrm{~s}^{4} \mathrm{D}-3 \mathrm{~d}^{4}\left({ }^{3} \mathrm{D}\right) 4 \mathrm{p}^{4} \mathrm{D}^{o}$ & 2846.26 & 0.90349 & -0.49312 & -0.00082 & 0.9999991 \\
$3 \mathrm{~d}^{4}\left({ }^{5} \mathrm{D}\right) 4 \mathrm{~s}^{6} \mathrm{D}-3 \mathrm{~d}^{4}\left({ }^{5} \mathrm{D}\right) 4 \mathrm{p}^{6} \mathrm{~F}^{o}$ & 2850.70 & 0.97414 & -0.52729 & 0.00325 & 0.9999963 \\
\hline \hline
\end{tabular}

We compared in Table 3 our results with those obtained experimentally by Aguilera et al. (2014). For spectral line widths within a multiplet, we used the formula:

$$
W_{\text {line }}=\left(\frac{\lambda_{\text {line }}}{\lambda_{\text {Mult }}}\right)^{2} W_{\text {Mult }}
$$

where $W_{\text {line }}$ and $\lambda_{\text {line }}$ are for the particular line within the multiplet; $W_{\text {Mult }}$ and $\lambda_{\text {Mult }}$ are the values for the multiplet.

Table 3. Comparison of our MSE Stark width calculations $\mathrm{W}_{M S E}$ with experimental widths $\mathrm{W}_{\text {Agl }}$ from Aguilera et al. (2014). Lines are sorted by wavelength.

\begin{tabular}{|c|c|c|c|c|c|}
\hline Transition & Multiplet & $\lambda(\AA)$ & $\mathrm{W}_{A g l}$ & $\mathrm{~W}_{M S E}$ & $\frac{W_{A g l}}{W_{M S E}}$ \\
\hline $3 d^{4}\left(a^{1} G\right) 4 s-3 d^{4}\left(a^{1} G\right) 4 p$ & $\mathrm{c}^{2} \mathrm{G} \quad \mathrm{w}^{2} \mathrm{G}^{\mathrm{o}}$ & 2774.43 & $6.96 \mathrm{E}-02$ & $6.30 \mathrm{E}-02$ & 0.91 \\
\hline $3 d^{4}\left({ }^{5} \mathrm{D}\right) 4 \mathrm{~s}-3 \mathrm{~d}^{4}\left({ }^{5} \mathrm{D}\right) 4 \mathrm{p}$ & $\mathrm{a}^{6} \mathrm{D} \quad \mathrm{z}^{6} \mathrm{~F}^{o}$ & 2835.63 & 6.91E-02 & $5.00 \mathrm{E}-02$ & 0.72 \\
\hline $3 d^{4}\left({ }^{3} \mathrm{D}\right) 4 \mathrm{~s}-3 \mathrm{~d}^{4}\left({ }^{3} \mathrm{D}\right) 4 \mathrm{p}$ & $\mathrm{c}^{4} \mathrm{D} \quad \mathrm{w}{ }^{4} \mathrm{D}^{o}$ & 2838.78 & $6.41 \mathrm{E}-02$ & $5.50 \mathrm{E}-02$ & 0.86 \\
\hline $3 d^{4}\left({ }^{5} \mathrm{D}\right) 4 \mathrm{~s}-3 \mathrm{~d}^{4}\left({ }^{5} \mathrm{D}\right) 4 \mathrm{p}$ & $\mathrm{a}^{6} \mathrm{D} \mathrm{z}^{6} \mathrm{~F}^{o}$ & 2843.25 & $6.94 \mathrm{E}-02$ & 5.10E-02 & 0.73 \\
\hline $3 \mathrm{~d}^{4}\left({ }^{5} \mathrm{D}\right) 4 \mathrm{~s}-3 \mathrm{~d}^{4}\left({ }^{5} \mathrm{D}\right) 4 \mathrm{p}$ & $\mathrm{a}^{6} \mathrm{D} \quad \mathrm{z}^{6} \mathrm{~F}^{o}$ & 2849.83 & $6.98 \mathrm{E}-02$ & $5.10 \mathrm{E}-02$ & 0.73 \\
\hline $3 d^{4}\left({ }^{5} \mathrm{D}\right) 4 \mathrm{~s}-3 \mathrm{~d}^{4}\left({ }^{5} \mathrm{D}\right) 4 \mathrm{p}$ & $\mathrm{a}^{6} \mathrm{D} \mathrm{z}^{6} \mathrm{~F}^{o}$ & 2855.67 & $7.00 \mathrm{E}-02$ & $5.00 \mathrm{E}-02$ & 0.71 \\
\hline $3 \mathrm{~d}^{4}\left({ }^{5} \mathrm{D}\right) 4 \mathrm{~s}-3 \mathrm{~d}^{4}\left({ }^{5} \mathrm{D}\right) 4 \mathrm{p}$ & $\mathrm{a}^{6} \mathrm{D}$ z ${ }^{6} \mathrm{~F}^{\circ}$ & 2860.93 & $7.03 \mathrm{E}-02$ & $5.00 \mathrm{E}-02$ & 0.71 \\
\hline $3 d^{4}\left({ }^{5} \mathrm{D}\right) 4 \mathrm{~s}-3 \mathrm{~d}^{4}\left({ }^{5} \mathrm{D}\right) 4 \mathrm{p}$ & $\mathrm{a}^{6} \mathrm{D} \mathrm{z}^{6} \mathrm{~F}^{\circ}$ & 2862.57 & $7.04 \mathrm{E}-02$ & 4.70E-02 & 0.67 \\
\hline $3 d^{4}\left({ }^{3} G\right) 4 s-3 d^{4}\left({ }^{3} G\right) 4 p$ & $\mathrm{~b}^{2} \mathrm{G} \times{ }^{2} \mathrm{G}^{o}$ & 2927.08 & $8.27 \mathrm{E}-02$ & $7.90 \mathrm{E}-02$ & 0.96 \\
\hline $3 d^{4}\left({ }^{3} \mathrm{P}\right) 4 \mathrm{~s}-3 \mathrm{~d}^{4}\left(\mathrm{a}^{3} \mathrm{P}\right) 4 \mathrm{p}$ & $\mathrm{b}^{4} \mathrm{P} \quad \mathrm{y}{ }^{4} \mathrm{D}^{\circ}$ & 2930.85 & $7.50 \mathrm{E}-02$ & $6.60 \mathrm{E}-02$ & 0.88 \\
\hline $3 d^{4}\left({ }^{3} \mathrm{P}\right) 4 \mathrm{~s}-3 \mathrm{~d}^{4}\left(\mathrm{a}^{3} \mathrm{P}\right) 4 \mathrm{p}$ & $\mathrm{b}^{4} \mathrm{P} \quad \mathrm{y}{ }^{4} \mathrm{D}^{o}$ & 2935.13 & $7.53 \mathrm{E}-02$ & $6.60 \mathrm{E}-02$ & 0.88 \\
\hline $3 d^{4}\left({ }^{3} \mathrm{P}\right) 4 \mathrm{~s}-3 \mathrm{~d}^{4}\left(\mathrm{a}^{3} \mathrm{P}\right) 4 \mathrm{p}$ & $\mathrm{b}^{4} \mathrm{P} \quad \mathrm{y}{ }^{4} \mathrm{D}^{o}$ & 2961.72 & $7.66 \mathrm{E}-02$ & $6.20 \mathrm{E}-02$ & 0.81 \\
\hline $3 \mathrm{~d}^{4}\left({ }^{3} \mathrm{H}\right) 4 \mathrm{~s}-3 \mathrm{~d}^{4}\left({ }^{3} \mathrm{H}\right) 4 \mathrm{p}$ & $\mathrm{a}^{4}{ }^{4} \mathrm{H} \quad{ }^{4} \mathrm{H}^{o}$ & 2971.90 & $7.71 \mathrm{E}-02$ & $7.10 \mathrm{E}-02$ & 0.92 \\
\hline $3 d^{4}\left({ }^{3} \mathrm{P}\right) 4 \mathrm{~s}-3 \mathrm{~d}^{4}\left(\mathrm{a}^{3} \mathrm{P}\right) 4 \mathrm{p}$ & $\mathrm{b}^{4} \mathrm{P} \quad \mathrm{y}{ }^{4} \mathrm{D}^{\circ}$ & 2976.71 & $7.74 \mathrm{E}-02$ & $6.00 \mathrm{E}-02$ & 0.78 \\
\hline $3 \mathrm{~d}^{4}\left({ }^{3} \mathrm{H}\right) 4 \mathrm{~s}-3 \mathrm{~d}^{4}\left({ }^{3} \mathrm{H}\right) 4 \mathrm{p}$ & $\mathrm{a}^{4} \mathrm{H} \quad \mathrm{z}^{4} \mathrm{H}^{\circ}$ & 2979.74 & $7.75 \mathrm{E}-02$ & $7.00 \mathrm{E}-02$ & 0.90 \\
\hline $3 d^{4}\left({ }^{3} \mathrm{H}\right) 4 \mathrm{~s}-3 \mathrm{~d}^{4}\left({ }^{3} \mathrm{H}\right) 4 \mathrm{p}$ & $\mathrm{a}^{4} \mathrm{H} \quad \mathrm{z}{ }^{4} \mathrm{H}^{o}$ & 2989.19 & $7.80 \mathrm{E}-02$ & $6.80 \mathrm{E}-02$ & 0.87 \\
\hline $3 d^{4}\left({ }^{3} F\right) 4 s-3 d^{4}\left(a^{3} F\right) 4 p$ & $\mathrm{~b}^{2} \mathrm{~F} \quad \mathrm{z}^{2} \mathrm{~F}^{o}$ & 3028.12 & $8.55 \mathrm{E}-02$ & $7.70 \mathrm{E}-02$ & 0.90 \\
\hline $3 d^{4}\left({ }^{3} \mathrm{P}\right) 4 \mathrm{~s}-3 \mathrm{~d}^{4}\left(\mathrm{a}^{3} \mathrm{P}\right) 4 \mathrm{p}$ & $\mathrm{a}^{2} \mathrm{P} \mathrm{z}^{2} \mathrm{P}^{o}$ & 3172.07 & $9.47 \mathrm{E}-02$ & $7.80 \mathrm{E}-02$ & 0.82 \\
\hline
\end{tabular}

We obtain for all the considered lines 0.82 as a mean value of the ratio $\frac{W_{A g l}}{W_{M S E}}$ with a standard deviation of $\mathrm{s}=0.088$, so the calculated values are approximately $20 \%$ higher than the experimental ones. 


\section{Conclusions}

We calculated Stark electron-impact widths using the MSE method for a range of temperatures from $5000 \mathrm{~K}$ to $50000 \mathrm{~K}$ and for an electron density of $10^{17}$ $\mathrm{cm}^{-3}$. These new eight Cr II Stark widths calculated in this work, for the lines were other theoretical Stark broadening data are missing, are of interest for stellar plasma, laboratory plasma diagnostics, laser produced plasma, as well as for analysis and modeling of stellar atmospheres. An extension of the Stark broadening calculations to other lines of Cr II will be an interesting future work and also applications of the atomic and spectroscopic data of these lines to typical stars where Stark broadening is an important mechanism in their atmospheres.

Acknowledgements. This project was funded by the National Plan for Science, Technology and Innovation (MAARIFAH), King Abdulaziz City for Science and Technology, Kingdom of Saudi Arabia, Award Number (12-MAT2879-02).

\section{References}

Aguilera, J.A., Aragón, C., and Manrique, J.: Experimental Stark widths and shifts of Cr II spectral lines 2014, Mon. Not. R. Astron. Soc. 438, 841.

Bates, D.R., and Damgaard, A.: The Calculation of the Absolute Strengths of Spectral Lines 1949, Phil. Transact. Roy. Soc. London A 242, 101.

Cowley, C.R.: An approximate Stark broadening formula for use in spectrum synthesis 1971, Observatory 91, 139.

Dimitrijević, M., and Chougule, A.: Stark Broadening of Cr III Spectral Lines: DO White Dwarfs 2018, Atoms 6, 15.

Dimitrijević, M.S., and Konjević, N.: Stark widths of doubly- and triply-ionized atom lines. 1980, J. Quant. Spectrosc. Radiat. Transfer 24, 451.

Dimitrijević, M.S., Ryabchikova, T., Popović, L.Č., Shulyak, D., and Khan, S.: On the influence of Stark broadening on Cr I lines in stellar atmospheres 2005, Astron. Astrophys. 435, 1191.

Dimitrijević, M.S., Ryabchikova, T., Simić, Z., Popović, L.Č., and Dačić, M.: The influence of Stark broadening on Cr II spectral line shapes in stellar atmospheres 2007, Astron. Astrophys. 469, 681.

Dimitrijević, M.S., Simić, Z., and Sahal-Bréchot, S.: On the Stark broadening of Cr VI spectral lines in astrophysical plasma 2017, J. Phys. Conf. Series, 012021.

Dubernet, M.L., et al.: The virtual atomic and molecular data centre (VAMDC) consortium 2016, J. Phys. B: At. Mol. Phys. 49, 074003.

Dufour, P., Ben Nessib, N., Sahal-Bréchot, S., and Dimitrijević, M.S.: Stark Broadening of Carbon and Oxygen Lines in Hot DQ White Dwarf Stars: Recent Results and Applications 2011, Balt. Astron. 20, 511. 
Griem, H.R.: Semiempirical Formulas for the Electron-Impact Widths and Shifts of Isolated Ion Lines in Plasmas 1968, Phys. Rev. 165, 258.

Hamdi, R., Ben Nessib, N., Milovanović, N., Popović, L.Č., Dimitrijević, M.S., and Sahal-Bréchot, S.: Atomic data and electron-impact broadening effect in DO white dwarf atmospheres: SiVI 2008, Mon. Not. R. Astron. Soc. 387, 871.

Hamdi, R., Ben Nessib, N., Sahal-Bréchot, S., and Dimitrijević, M.S.: Stark widths of Ar III spectral lines in the atmospheres of subdwarf B stars 2014, Adv. Space Res. $\mathbf{5 4}, 1223$.

Kramida, A., Yu. Ralchenko, Reader, J., \& and NIST ASD Team. 2018, NIST Atomic Spectra Database (ver. 5.6.1), [Online]. Available: https://physics.nist.gov/asd [2019, April 18]. National Institute of Standards and Technology, Gaithersburg, MD.

Majlinger, Z., Simić, Z., and Dimitrijević, M.S.: Stark broadening of Zr IV spectral lines in the atmospheres of chemically peculiar stars 2017, Mon. Not. R. Astron. Soc. 470, 1911.

Moreau, N., Zwolf, C.-M., Ba, Y.-A., Richard, C., Boudon, V., and Dubernet, M.-L.: The VAMDC Portal as a Major Enabler of Atomic and Molecular Data Citation 2018, Galaxies 6, 105.

Oertel, G.K., and Shomo, L.P.: Tables for the Calculation of Radial Multipole Matrix Elements by the Coulomb Approximation. 1968, Astrophys. J., Suppl. Ser. 16, 175.

Sahal-Bréchot, S., Dimitrijević, M.S., and Ben Nessib, N.: Comparisons and Comments on Electron and Ion Impact Profiles of Spectral Lines 2011, Balt. Astron. 20, 523.

Sahal-Bréchot, S., Dimitrijević, M., and Ben Nessib, N.: 2014, Atoms 2, 225.

Sahal-Bréchot, S., Dimitrijević, M.S., Moreau, N., and Ben Nessib, N.: The STARK-B database VAMDC node: a repository for spectral line broadening and shifts due to collisions with charged particles 2015, Phys. Scr. 90, 054008.

Simić, Z., Dimitrijević, M.S., Popović, L.Č., and Dačić, M.D.: Stark broadening parameters for $\mathrm{Cu}$ III, Zn III and Se III lines in laboratory and stellar plasma 2006, New Astron. 12, 187.

Simić, Z., Dimitrijević, M.S., and Sahal-Bréchot, S.: Stark broadening of resonant Cr II $3 \mathrm{~d}^{5}-3 \mathrm{~d}^{4} 4 \mathrm{p}$ spectral lines in hot stellar atmospheres 2013, Mon. Not. R. Astron. Soc. 432, 2247.

van Regemorter, H., Binh Dy, H., and Prudhomme, M.: Radial transition integrals involving low or high effective quantum numbers in the Coulomb approximation 1979, J. Phys. B: At. Mol. Phys. 12, 1053. 